\title{
New approaches to predicting the risk of sudden death
}

\author{
Authors: Elijah Behr ${ }^{A}$ and Bode Ensam ${ }^{B}$
}

In this review article, we will explore some of the contemporary methods for predicting sudden cardiac death (SCD). These include experimental methods yet to be adopted in the clinical setting, and methods that have been extrapolated from observational data in those with a history of SCD. We will discuss how these relate to the different aetiologies and disease processes. We will also explore how these may be used in the clinical setting to decide on management.

\section{Introduction}

Determining an individual's risk of sudden death from lifethreatening cardiac arrhythmias (sudden cardiac death (SCD)) remains a challenging task. An understanding of high-risk features allows clinicians to identify those who will benefit most from risk-modifying therapies or other forms of treatment.

Secondary prevention in patients following a life-threatening arrhythmia can significantly reduce cardiovascular event rates. However, primary prevention in patients with acquired cardiac disease still relies heavily on impairment of left ventricular function. ${ }^{1}$ Most individuals with inherited cardiac conditions (ICCs) may display evidence of the condition but remain asymptomatic, yet the risk of SCD in these individuals is of paramount concern, especially in the absence of structural heart disease that may carry its own mortality. Large-scale evidence-based studies for clinicians to draw on are lacking.

Furthermore, the only definitive treatment currently available across all acquired and genetic disease entities in the prevention of SCD in high-risk patients is the implantable cardioverter defibrillator (ICD). This, however, has an enormous physical and psychological impact on the recipient and the decision to implant must be well thought out and justified robustly with objective evidence of risk.

Therefore, a number of methods to risk stratify these individuals are employed that may be specific to the disease process, whereas others may be of use across a spectrum of disease processes, acquired and genetic.

In this update review article, we will concentrate largely on recent developments in the assessment of risk of SCD and

Authors: A senior lecturer and honorary consultant cardiologist, Cardiovascular Research Unit, St George's University of London, London, UK; ${ }^{B}$ cardiology registrar and cardiology research fellow in inherited heart disease, Cardiovascular Research Unit, St George's University of London, London, UK discuss the prognostic impact these may have. While there is a focus on ICCs as exemplars of these techniques we will also reflect on the role of these approaches in acquired heart disease. Some of these methods are yet to withstand robust clinical validation and are of experimental and research value, whereas others are of potential use in current clinical practice. For practical utility most are non-invasive, although we also explore the role for invasive electrophysiological studies.

\section{Integrating old risk factors}

A novel risk calculator based on existing established and readily available risk factors offers one approach for extracting greater predictive accuracy. This has been undertaken for hypertrophic cardiomyopathy (HCM) and adopted early by the European Society of Cardiology. ${ }^{2}$

\section{Electrocardiogram markers of ventricular repolarisation}

The QT interval is the commonly used measure of ventricular repolarisation time; prolongation of the QT interval is associated with an increased risk of SCD. However, in most population-based studies, no consistent association has been found between QT interval prolongation and total or cardiovascular mortality, thus limiting its use as a clinical marker of risk. ${ }^{3}$ A number of other electrocardiogram (ECG) markers of ventricular repolarisation have been suggested as predictors for the development of ventricular arrhythmias in a variety of disease processes. ${ }^{4}$

\section{Tpeak-end interval}

More recently, however, the Tpeak-end (Tp-end) interval interval, the time between the peak and the end of the $\mathrm{T}$ wave proposed to represent transmural dispersion of ventricular repolarisation, has been found to predict the risk of malignant cardiac arrhythmias in a number of clinical settings (Fig 1). ${ }^{5}$ In 235 patients with Brugada syndrome $(\mathrm{BrS})$, Tp-end was significantly increased in those presenting with malignant arrhythmias or syncope compared with asymptomatic carriers. $^{6}$

In addition, both Tpeak-end and Tpeak-end/QT ratio have been examined during acute ST elevation myocardial infarction (STEMI). Tp-end was found to be significantly prolonged in STEMI compared with healthy individuals. A Tpeak-end $>0.1$ and Tpeak-end/QT ratio $>0.3$ predict malignant ventricular arrhythmias within 24 hours of STEMI. ${ }^{7}$ 


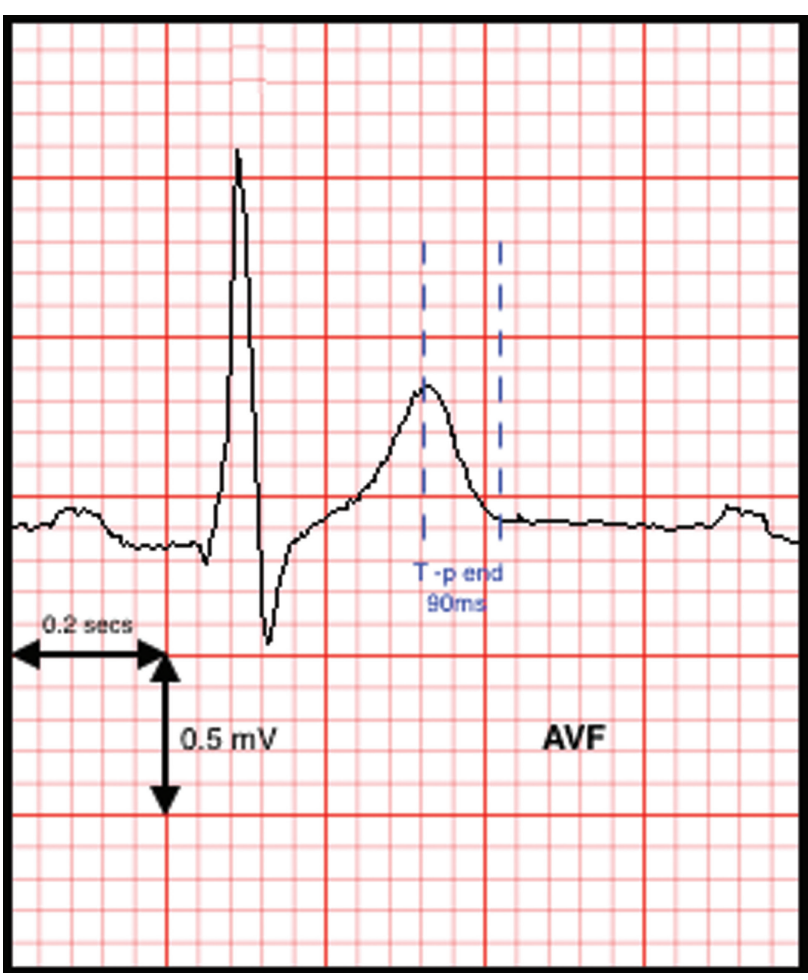

Fig 1. Tpeak - Tend interval (Tp-end).

\section{Microvolt T-wave alternans}

Another measure, T-wave alternans, is the beat-to-beat variation in the T-wave amplitude, and is an indicator of instability of repolarisation associated with impending arrhythmic risk

\section{Key points}

Clinical symptoms remain the most consistent means of determining risk of future events.

In the absence of symptoms, identification of high-risk features is paramount in order to determine those that might benefit from implantable cardiac defibrillator therapy.

With annual event rates being low in those with inherited cardiac disease, it is difficult to identify non-clinical markers that sufficiently and consistently separate high- and low-risk groups.

Novel electrocardiographic risk predictors aim to identify subtle abnormalities in repolarisation and depolarisation. However, with varying levels of sensitivity and specificity, consistent incorporation into clinical practice is difficult.

A risk prediction model that incorporates multiple parameters, including electrocardiogram data, structural information and genetics that can be used across a variety of diseases is likely to be the ideal model of the future.

KEYWORDS: Sudden cardiac death, ventricular arrhythmia, inherited heart disease and ischaemic heart disease described initially in the long-QT syndrome ${ }^{8}$ but applicable to other disorders. Microvolt T-wave alternans (MTWA) measures T-wave alternans that are undetectable visually.

In the Alternans Before Cardioverter Defibrillator (ABCD) study, ${ }^{9}$ MTWA was compared to invasive electrophysiological testing (EPS), in its ability to predict the risk of ventricle dysrhythmia in those with ischaemic cardiomyopathy (left ventricular ejection fraction $(\mathrm{LVEF})<40 \%$ ). Subjects underwent insertion of an ICD if either parameter was positive. At 1 year, positive and negative predictive values for MTWA (9 and $95 \%$, respectively) were comparable to EPS (11 and 95\%, respectively).

The REFINE trial demonstrated increased event rates in patients with MTWA in the sub-acute phase post myocardial infarction (MI). ${ }^{10}$ Merchant et al ${ }^{11}$ studied over 2,800 patients without a history of cardiac arrest or ventricular arrhythmias. MTWA was positive in 30\%, negative in 56\% and indeterminate in $14 \%$. In patients with LVEF $<35 \%$ and negative MTWA, annual event rates were low at $0.9 \%$, while event rates were significantly higher in the positive and indeterminate groups, 4.0 and $4.6 \%$, respectively. Patients with an LVEF of $>35 \%$ and a positive MTWA test were at increased risk of SCD: $3.0 \%$ compared with $0.3 \%$ in MTWA negative and indeterminate groups.

While intriguing, MTWA and Tp-end remain to be validated in routine clinical use as a number of limitations exist. For example, manual methods of measuring T-wave amplitudes and durations are inaccurate and, thus, automated methods have been developed; however, the computerised methods differ between manufacturer and this is likely to produce variability in reporting. Additionally, the range of normal values for Tpend and Tp-end/QT, which is likely to vary greatly, are yet to be robustly defined or explored in normal subjects.

Large prospective studies are required to evaluate the usefulness of these methods in different clinical entities given that the annual event rate can be extremely low.

Other potential measures of repolarisation include QTinterval dispersion, QT variability and QRST angle. As yet, none have been implemented in clinical practice because of a lack of robust validation. A review article by Tikkanen et al ${ }^{12}$ and work by Picarillo et al ${ }^{13}$ explore these additional markers in more detail.

\section{Early repolarisation}

Early repolarisation (ER) is currently defined as elevation of the QRS-ST junction (J point) in at least two leads in the inferior or lateral distribution. Elevation must be at least $1 \mathrm{~mm}(0.1 \mathrm{mV})$ above the baseline level (Fig 2a). This may manifest as slurring or notching of the QRS. It may be present in up to $15 \%$ of the population with a higher prevalence in young men, athletes and those of African descent. ${ }^{14}$

Previously, ER was thought to be a benign feature on the 12lead ECG but was linked to idiopathic ventricular fibrillation in 2008 by Haissaguerre et al. In 206 survivors of cardiac arrest from ventricular fibrillation, ER was demonstrated in $31 \%$. Additionally, recurrent VF was more common in those with ER. ${ }^{15}$

Further work by Tikkanen et al ${ }^{16}$ looked at the long-term outcome associated with ER in a cohort of over 10,000 middleaged Finnish subjects with a mean follow-up of $30 \pm 11$ years. 


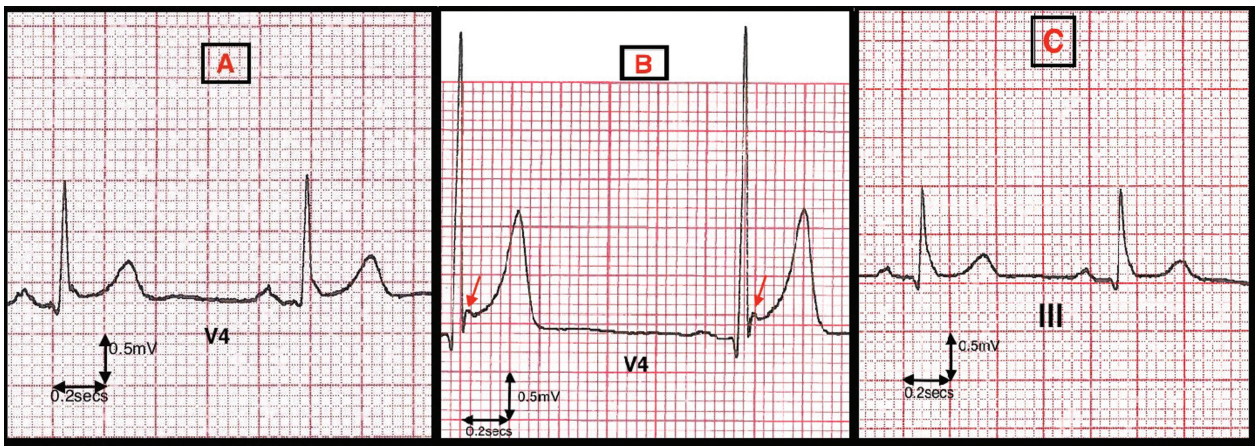

Fig 2. A - Typical J point elevation; $\mathrm{B}$ - upsloping ascending with notching (arrowed); C horizontal descending.

ER was demonstrated in 5.8\%. Inferior J-point elevation of $>0.1 \mathrm{mV}$ was associated with an increase in risk of cardiac death (relative risk (RR) 1.28; $\mathrm{p}=0.03$ ). J-point elevation $>0.2$ $\mathrm{mV}$ had a markedly elevated risk of cardiac death (RR 2.98; $p<0.001)$ and from arrhythmia (RR 2.92; $p=0.01)$. The Finnish group further stratified ER risk according to ST-segment morphology: ${ }^{17}$ upsloping ascending (Fig 2B) and horizontal descending (Fig 2C). The former appeared to have a benign association while the latter showed association with arrhythmic risk (hazard ratio (HR) 1.43; 95\% confidence interval 1.05-1.94). The clinical relevance of ER in the general population is low; however, given the high prevalence and low absolute risk, it should not be used as a clinical marker of risk in this context. ${ }^{14}$

In patients with an existing substrate for malignant arrhythmia, ER may be of greater clinical utility. In $\mathrm{BrS}$ for example, one study observed that $10-15 \%$ of patients had infero-lateral ER accompanied by an 11-fold increase in risk of VF. ${ }^{18}$ In addition, Chan et al ${ }^{19}$ investigated ER in a cohort of 59 arrhythmogenic right ventricular cardiomyopathy (ARVC) patients, 64\% demonstrating ER in the inferior leads who had a higher incidence of VF or aborted SCD. In acute STEMI, ER has been found to be associated with a significant increase in risk of ventricular tachyarrhythmia in the acute post infarction phase ( $<72$ hours), irrespective of left ventricular function and level of cardiac enzyme rise. ${ }^{20,21}$ In patients with known chronic coronary artery disease, ventricular arrhythmia has been found to be more common in those with inferior early repolarisation; additionally, the presence of associated notching was also strongly associated with risk. ${ }^{22}$ Further validation is required before ER can be employed in this setting.

\section{ECG markers of ventricular depolarisation}

The QRS duration as a readily available marker of ventricular depolarisation has also been associated with the risk of SCD in the general population. ${ }^{23,24}$ Patients with prior MI and/or heart failure with a QRS duration $>120 \mathrm{~ms}$ and/or left bundle branch block may be indicated for cardiac resynchronisation therapy alongside ICD therapy depending on symptoms and ejection fraction. ${ }^{1}$ The following other markers have been explored for additive value.

\section{Late ventricular potentials and the signal averaged ECG}

Late ventricular potentials are abnormal low-amplitude signals in the terminal portion of the QRS complex reflecting delayed depolarisation in the ventricles. This suggests the presence of potential substrates for ventricular arrhythmia. Late potentials cannot, however, be visualised on a standard ECG recording requiring a signal averaged ECG (SAECG) being acquired over a prolonged period (7-10 minutes). During this period, a number of QRS complexes are collected from orthogonal ECG leads ( $x, y$ and $z$ ) with wide-frequency filters and averaged to reduce interference due to artefact. These signals can then be processed using ECG analysing software (Fig 3).

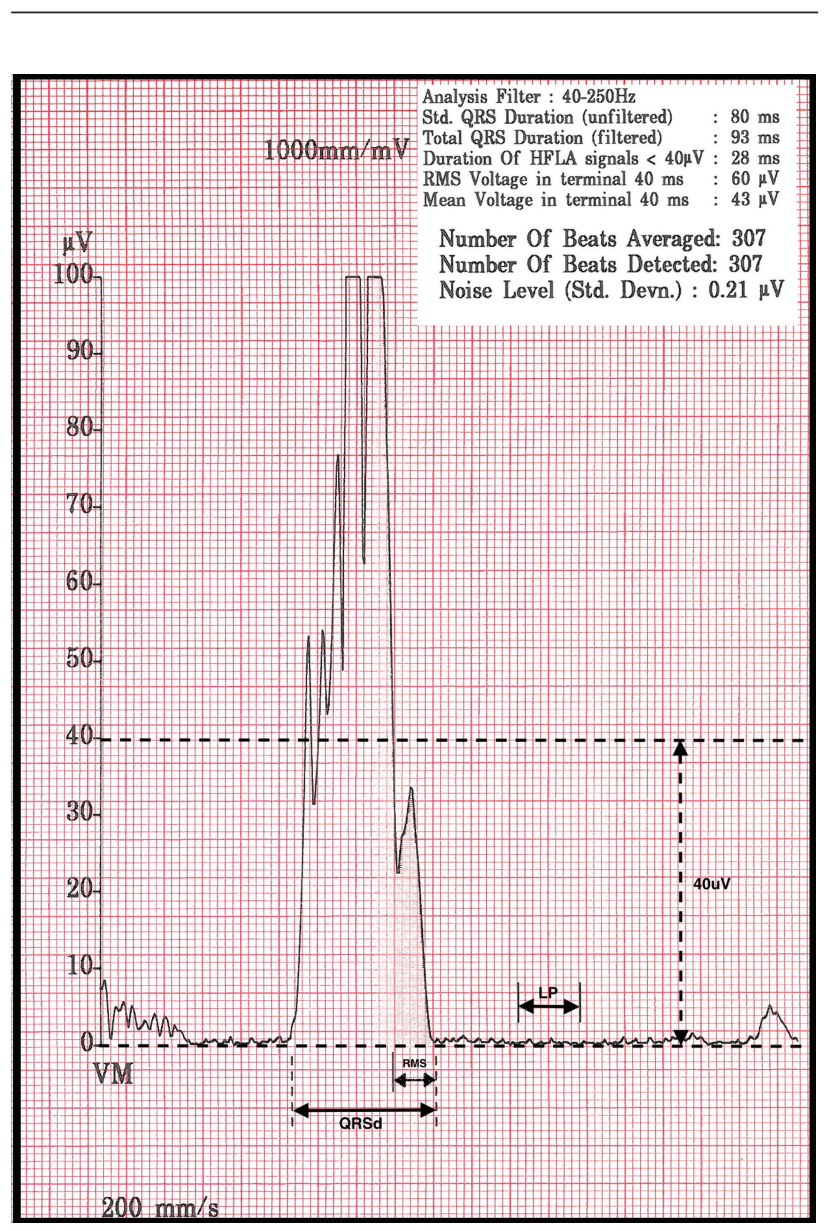

Fig 3. Signal-averaged electrocardiogram. $L P=$ late potentials; $Q R S d=$ total QRS duration; RMS = root mean square in the terminal $40 \mathrm{~ms}$ of QRS. 


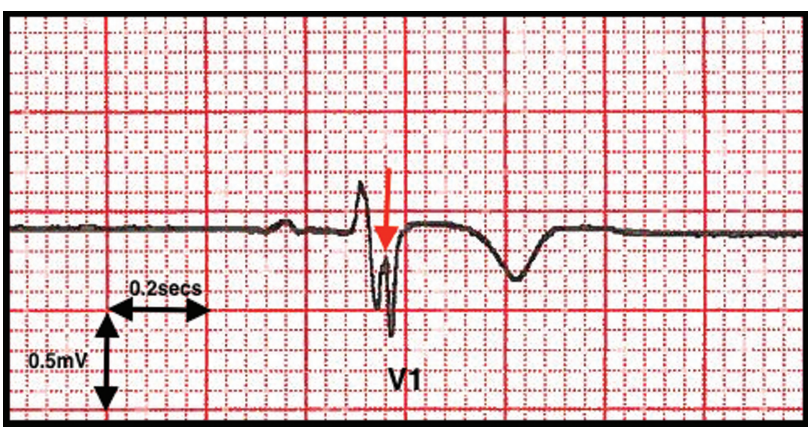

Fig 4. Notching within the $\mathrm{S}$ wave.

The presence of late potentials is a strong predictor of ventricular arrhythmias in a number of conditions, including post-MI, dilated cardiomyopathy and arrhythmogenic cardiomyopathy, ${ }^{25}$ and is a diagnostic tool in arrhythmogenic right ventricular cardiomyopathy. ${ }^{26}$ A number of small contemporary studies have attempted to evaluate the use of the SAECG in the BrS. There is an increased risk of ventricular fibrillation in those patients in whom late potentials are detected, although an old meta-analysis has suggested that in multivariable analysis the association may be lost. ${ }^{27}$

Unfortunately, while the SAECG is a non-invasive, quantitative tool that can be repeated over time, it requires a specialist ECG machine or plug-in that most centres do not use routinely. It therefore remains an underutilised tool.

\section{QRS fragmentation}

Fragmentation of the QRS complex (f-QRS) refers to slurring and notching within the QRS morphology and has been correlated with the presence of myocardial scar and fibrosis (Figs 4 and 5). ${ }^{28} \mathrm{f}$-QRS has been demonstrated to predict cardiac arrhythmias in conditions such as ARVC, acute MI, dilated cardiomyopathy and infiltrative conditions such as sarcoidosis. $^{28}$
The PRELUDE study ${ }^{29}$ evaluated 308 patients with BrS without a history of cardiac arrest. This concluded that patients with $\mathrm{f}-\mathrm{QRS}$ had a nine times greater risk of developing VF. Tokioka et $_{\mathrm{al}} \mathrm{l}^{30}$ then examined the $\mathrm{f}-\mathrm{QRS}$ and ER for predicting outcomes in BrS patients. They demonstrated that presence of both ER and f-QRS significantly increased the risks of cardiac arrhythmias compared with patients with $\mathrm{f}-\mathrm{QRS}$ alone or without either abnormality. The event rates in patients with neither abnormality were extremely low. At this time, guidelines on prophylactic device therapy do not indicate $\mathrm{f}-\mathrm{QRS}$ or ER as accepted markers for risk assessment. ${ }^{31}$ They may, however, offer value in risk stratification on a case-by-case basis.

\section{Markers of autonomic dysfunction}

\section{Heart rate variability and turbulence}

Heart rate variability (HRV) is the beat-to-beat variation in heart rate, this is influenced by autonomic effects on the sinoatrial node. The changes in the heart rate in response to a premature ventricular complex is termed the heart rate turbulence (HRT); there is a baroreflex-mediated influence on the sinoatrial node which results in a temporary increase in heart rate followed by a decrease back to the baseline. Impaired autonomic function, as measured by HRV and HRT, has been proven to predict adverse outcomes in clinical settings, including post-MI $\mathrm{M}^{32,33}$ and congestive cardiac failure. ${ }^{34}$ However, in a large meta-analysis of risk predictors in the nonischemic dilated cardiomyopathy group, it was demonstrated that the autonomic factors were not significant predictors of arrhythmic outcome, but had a high negative predictive accuracy $^{35}$. Much like MTWA, the limitations in uptake by Holter manufacturers and variable software packages have resulted in limited use except by specialist centers.

\section{Invasive programmed electrical stimulation}

Electrophysiological study for investigating the risk of sudden death was first used to test drug suppression of ventricular arrhythmias in cardiac arrest survivors. It was then utilised
Fig 5. Widespread QRS fragmentation in a patient with Brugada syndrome.

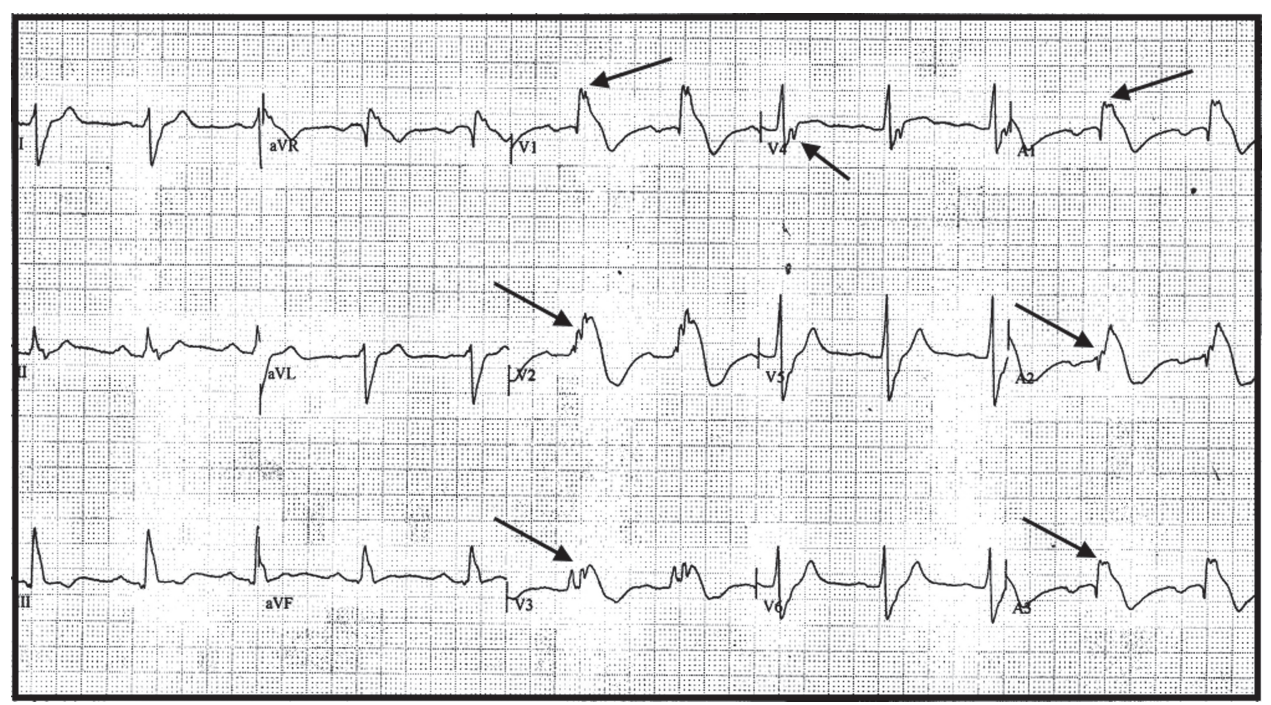


in risk stratification of post-infarct patients prior to ICD implantation. ${ }^{36}$ It quickly became apparent that inducibility of ventricular tachycardia was less effective a marker than ejection fraction alone. ${ }^{37,38}$ Inducibility of ventricular fibrillation was always thought to be non-specific and clinically unhelpful, especially in a structurally normal heart. ${ }^{39}$ Thus, while it has fallen out of fashion in other arenas, its role in prediction of future events in $\mathrm{BrS}$ is a topic of current debate. A variety of protocols have been employed involving stimulation at the right ventricular apex with or without additional pacing at the outflow tract and the use of a maximum of two or three extra stimuli with a minimum pacing interval of $200 \mathrm{~ms}$. A positive test has been defined as a sustained ventricular arrhythmia.

The Brugada brothers first proposed that inducibility of programmed electrical stimulation (PES) could predict SCD irrespective of presenting symptoms and ECG. ${ }^{40-42}$ Despite these findings, subsequent large multi-centered analysis failed to replicate these associations. The FINGER study, ${ }^{43}$ the largest European registry of $\mathrm{BrS}$ with over 1,029 consecutive patients, demonstrated in a multivariate analysis that inducibility was not an independent predictor of cardiac events. The PRELUDE study ${ }^{29}$ found no significant differences between those with inducible ventricular arrhythmia and those without. It did, however, highlight the value of QRS fragmentation and the ventricular effective refractory period (VERP) as two important risk predictors. VERP is the maximum pacing interval between extra stimuli at which the ventricle is refractory. A VERP $<200$ $\mathrm{ms}$ had a greater sensitivity and specificity at predicting events than inducibility, and was associated with a HR of 3.9. Despite the observed differences in the reliability of inducibility, a key factor in all studies is the link between the presence of high-risk clinical features and future events; with a spontaneous type-1 pattern and syncope being the stand out clinical determinants. Meta-analyses by Letsas et $a l^{44}$ and Sroubeck et $a l^{45}$ confirm these associations.

\section{Imaging}

\section{Cardiac magnetic resonance imaging}

Myocardial fibrosis and scar is a key substrate for ventricular arrhythmias regardless of aetiology. Contrast magnetic resonance imaging (MRI) using gadolinium is the gold standard imaging modality to identify the presence of fibrosis by detection of late gadolinium enhancement (LGE). ${ }^{46}$ Iles et $a l^{47}$ prospectively evaluated a cohort of 103 patients with nonischaemic or ischaemic cardiomyopathy eligible for primary prevention ICD. Among non-ischaemic group, the presence of fibrosis was associated with arrhythmic events during follow-up in one-third, whereas non-ischaemic patients without fibrosis remained arrhythmia free. While LGE is an independent risk factor for all-cause mortality, those with evidence of diffuse or mid-wall fibrosis have higher risk. ${ }^{48}$ The finding of LGE in HCM patients also appears to associate with risk of SCD and offers an additional risk factor to be taken into account in patient management. ${ }^{49}$

\section{ECG mapping}

ECG imaging is a non-invasive imaging modality, which reconstructs epicardial signals using body surface electrodes, mapping cardiac electrical excitation in relation to the heart's anatomy derived from high-resolution thoracic computerised tomography or MRI. This produces a digital image of epicardial electrograms and activation/repolarisation sequences. It has been proposed that ECG imaging could be used to identify repolarisation abnormalities, fractionated signals and lowamplitude electrograms that are potential substrates for malignant arrhythmias. ${ }^{50}$ This could be applicable to those with cardiomyopathy, post-MI or inherited cardiac conditions. There may also be a role in supporting ventricular tachycardia ablation. ${ }^{51}$ As yet, the role of ECG imaging in risk evaluation remains in research but it appears to offer a highly accurate, multi-dimensional assessment of arrhythmia characterisation and localisation, which sets it apart from other non-invasive ECG markers.

\section{Biomarkers and genomics}

Biomarkers for inflammation, neurohumoral activation and cardiac injury have been studied in patients with primary prevention ICDs to predict appropriate shocks (a surrogate marker for SCD) and all-cause mortality. ${ }^{52}$ PROSE-ICD ${ }^{52}$ investigated five potential biomarkers; C-reactive protein, interleukin-6, tumour necrosis factor- $\alpha$ receptor II, pro-brain natriuretic peptide (pro-BNP) and cardiac troponin T. All markers were associated with a significant increase in allcause mortality but only interleukin-6 had an association with predicting appropriate shock therapy.

Levin et $a l^{53}$ demonstrated that the risk of ventricular arrhythmias was significantly higher in those with elevated $\mathrm{N}$-terminal-proBNP (NT-proBNP) and BNP levels after adjustment for other risk factors. Whether this is purely a marker of suboptimal medical therapy or whether this can be used to aid ICD insertion, needs further evaluation.

Genetic testing in the setting of cardiac genetic disease, such as the long-QT syndrome and HCM, is clinically useful in predicting risk of sudden death in suspicious cases and relatives of index cases. ${ }^{54}$ While genotype alone has a minimal role in risk stratification at present, the presence of multiple mutations is associated with higher risk. ${ }^{55}$

The risk of SCD from acquired cardiac disease also has a genetic influence. In the AGNES study, cardiac arrest due to ventricular fibrillation following a first acute STEMI was three-fold more common in those with a family history for SCD than those without. ${ }^{56}$ Genome-wide studies associated this risk with the common genetic variant rs $2824292,{ }^{57}$ located close to a gene (CXADR) encoding for the coxsackie virus receptor. This appears to alter cardiac conduction in the presence of ischaemia. ${ }^{56,57}$ Multiple common genetic variants and their additive effects may also predict the risk of SCD. ${ }^{58}$

In general, however, genomic risk markers and biomarkers offer small increments in absolute risk level and the former are only applicable to specific ethnic populations. Their value when integrated with other clinical risk markers remains to be explored and their role is in research at this time.

\section{Conclusion}

Use of high-risk features enabling clinicians to identify those whose benefit from ICD therapy may outweigh the associated risks from the procedure and future device-related complications. However, current National Institute for Health 
and Care Excellence guidelines ${ }^{1}$ for ICD therapy are limited by conventional risk stratifiers.

Unfortunately, many of the markers identified in this review (and there are many others too numerous to include) are limited in their adoption in routine clinical activity by a number of factors: the need for additional equipment and/ or expense with variation in manufacturer specifications and software; limited robust validation in clinical settings; and small independent incremental value in risk assessment.

Regardless of the underlying disease, the most difficult group to stratify are those who are asymptomatic. Parameters extrapolated from survivors of cardiac arrest and an understanding of the natural progression of the disease type support risk stratification in the asymptomatic group, especially in rarer disorders. However, with some disease types having low annual events rates, $\mathrm{BrS}$ for example, it is difficult to identify non-clinical factors that separate high- and low-risk groups.

The time is now ripe for major prospective multimodality studies assessing novel risk markers in the general population and patients with coronary artery disease and heart failure. Multicentre registries with deep phenotyping of rare disorders will be required to establish new accurate management protocols. Only then will risk prediction be optimised for the individual and ICD therapy directed more accurately.

\section{References}

1 National Institute for Health and Care Excellence. Insertion of a subcutaneous implantable cardioverter defibrillator for prevention of sudden cardiac death NICE interventional procedure guidance [IPG454]. London: NICE, 2013.

2 O'Mahony C, Elliot PM, Pavlou M et al; Hypertrophic Cardiomyopathy Outcomes Investigators. A Novel clinical risk prediction model for sudden cardiac death in hypertrophic cardiomyopathy (HCM risk- SCD). Eur Heart J 2014;35:2010-20.

3 Straus SM, Kors JA, de Bruin ML et al. Prolonged QTc interval and risk of sudden cardiac death in a population of older adults. J Am Coll Cardiol 2006;47:362-7.

4 Gupta P, Patel C, Patel H et al (Tp-e)/QT ratio as an index of arrhythmogenesis. J Electrocardiol 2008;41:567-574.

5 Hevia C, Antzelevitch C, Tornés Bárzaga F et al. Tpeak-Tend and Tpeak-Tend dispersion as risk factors for ventricular tachycardia/ ventricular fibrillation in patients with the Brugada syndrome. J Am Coll Cardiol 2006;47:1828-34.

6 Maury P, Sacher F, Haïssaguerre M, et al. Increased Tpeak-Tend interval is highly and independently related to arrhythmic events in Brugada syndrome. Heart Rhythm 2015;7:029.

7 Shenthar J, Deora S, Rai M, et al. Prolonged Tpeak-end and Tpeak-end/QT ratio as predictors of malignant ventricular arrhythmias in the acute phase of ST-segment elevation myocardial infarction: a prospective case-control study. Heart Rhythm 2015;3:484-9.

8 Zareba W, Moss AJ, le Cessie S, et al. T wave alternans in idiopathic long QT syndrome. J Am Coll Cardiol 1994;7:1541-6.

9 Costantini O, Hohnloser SH, Kirk MM et al; ABCD Trial Investigators. The ABCD (Alternans Before Cardioverter Defibrillator) Trial: strategies using T-wave alternans to improve efficiency of sudden cardiac death prevention. J Am Coll Cardiol 2009; 10;53:471-9.

10 Exner DV, Kavanagh KM, Slawnych MP, et al. Noninvasive Risk Assessment Early After a Myocardial Infarction: The REFINE Study. J Am Coll Cardiol 2007;24:2275-84.

11 Merchant FM, Faisal M, Pedretti RF et al. Clinical utility of microvolt T-wave alternans testing in identifying patients at high or low risk of sudden cardiac death. Heart Rhythm 2012;8:1256-64.
12 Tikkanen JT, Kenttä T, Porthan K et al. Electrocardiographic T wave abnormalities and the risk of sudden cardiac death: the Finnish perspective. Ann Noninvasive Electrocardiol 2015;20:526-33.

13 Piccirillo G, Magrì D, Matera $\mathrm{S}$ et al. QT variability strongly predicts sudden cardiac death in asymptomatic subjects with mild or moderate left ventricular systolic dysfunction: a prospective study. Eur Heart J 2007;28:1344-50.

14 Bastiaenen R, Behr ER. Early repolarisation: controversies and clinical implications. Heart 2012;98:841-7.

15 Haissaguerre M, Derval N, Sacher F et al. Sudden cardiac arrest associated with early repolarization. N Engl J Med 2008;358:2016-23

16 Tikkanen JT, Anttonen O, Junttila MJ et al. Long-term outcome associated with early repolarization on electrocardiography. $N$ Engl J Med 2009;361:2529-37.

17 Tikkanen JT. Early repolarisation. Electrographic phenotypes associated with favourable long-term outcome. Circulation 2011 123:2666-73.

18 Takagi M, Aonuma K, Sekiguchi Y et al. The prognostic value of early repolarization (J wave) and ST-segment morphology after $\mathrm{J}$ wave in Brugada syndrome: multicenter study in Japan. Heart Rhythm 2013;10:533-9.

19 Chan CS, YJ2 Lin, Chang SL et al. Early repolarization of surface ECG predicts fatal ventricular arrhythmias in patients with arrhythmogenic right ventricular dysplasia/cardiomyopathy and symptomatic ventricular arrhythmias. Int J Cardiol 2015;197:300-5

20 Patel R.B, Ilkhanoff L, Ng J, Chokshi M et al. Clinical characteristics and prevalence of early repolarization associated with ventricular arrhythmias following acute ST-elevation myocardial infarction. Am J Cardiol 2012;110:615-20.

21 Rudic B, Veltmann C, Kuntz E et al. Early repolarization pattern is associated with ventricular fibrillation in patients with acute myocardial infarction. Heart Rhythm 2012;9:1295-300.

22 Patel RB, Ng J, Reddy V, Chokshi M et al. Early repolarization associated with ventricular arrhythmias in patients with chronic coronary artery disease Circulation 2010;3:489-95.

23 Aro AL, Anttonen O, Tikkanen JT et al. Intraventricular conduction delay in a standard 12-lead electrocardiogram as a predictor of mortality in the general population. Circulation 2011;4:704-10.

24 Kurl S, Mäkikallio TH, Rautaharju P et al. Duration of QRS complex in resting electrocardiogram is a predictor of sudden cardiac death in men. Circulation 2012;125:2588-94.

25 Steinberg JS, Berbari EJ. The signal-averaged electrocardiogram: Update on clinical applications. J Cardiovasc Electrophysiol 1996;7:972-88.

26 Marcus FI, McKenna WJ, Sherrill D et al. Diagnosis of right ventricular cardiomyopathy/ dysplasia. Proposed modification of the Task Force Criteria. Circulation 2012;121:1533-41.

27 Li A, Behr ER. Brugada syndrome: an update. Future Cardiol 2013;9:253-71.

28 Chatterjee S, Changawala N. Fragmented QRS Complex: a novel marker of cardiovascular disease. Clinical Cardiol 2010;33:68-71.

29 Priori SG, Gasparini M, Napolitano C et al. Risk stratification in Brugada syndrome: results of the PRELUDE (PRogrammed ELectrical stimUlation preDictive valuE) registry. J Am Coll Cardiol 2012;59:37-45.

30 Tokioka K, Morita H, Morita H et al. Electrocardiographic parameters and fatal arrhythmic events in patients with Brugada syndrome: combination of depolarization and repolarization abnormalities. J Am Coll Cardiol 2014:63:2131-8.

31 Priori SG, Blomström-Lundqvist C, Mazzanti A et al. ESC Guidelines for the management of patients with ventricular arrhythmias and the prevention of sudden cardiac death: The Task Force for the Management of Patients with Ventricular Arrhythmias and the Prevention of Sudden Cardiac Death of the European Society of Cardiology (ESC). Endorsed by: Association for European Paediatric and Congenital Cardiology (AEPC). Eur Heart J 2015;36:2793-867. 
32 Bauer A, Kantelhardt JW, Schmidt G, et al Deceleration capacity of heart rate as a predictor of mortality after myocardial infarction: cohort study. Lancet 2006; 367:1674-81.

33 Schmidt G, Barthel P, Schneider R, et al. Risk stratification after acute myocardial infarction by heart rate turbulence. Circulation 2003;108:1221-6.

34 Cygankiewicz I, Zareba W, Vazquez R et al Heart rate turbulence predicts all-cause mortality and sudden death in congestive heart failure patients. Heart Rhythm 2008;5:1095-102.

35 Goldberger J, Subačius H, et al. Sudden cardiac death risk stratification in patients with non ischemic dilated cardiomyopathy. J Am Coll Cardiol 2014;63: 1879-89.

36 Prystowsky EN, Nisam S. Prophylactic implantable cardioverter defibrillator trials: MUSTT, MADIT, and beyond. Multicenter Unsustained Tachycardia Trial. Multicenter Automatic Defibrillator Implantation Trial. Am J Cardiol 2000;86:1214-5.

37 Moss A, Zareba W, Hall W, et al; on behalf of the Multicenter Automatic Defibrillator Implantation Trial II Investigators. Prophylactic implantation of a defibrillator in patients with myocardial infarction and reduced ejection fraction. $N$ Engl J Med 2002;346:877-83.

38 Bardy GH, Lee KL, Mark DB et al; on behalf of the Sudden Cardiac Death in Heart Failure Trial (SCD-HeFT) Investigators. Amiodarone or an implantable cardioverter-defibrillator for congestive heart failure. Am J Cardiol 2005;352:225-37.

39 Brugada P, Abdollah H, Heddle B, Wellens HJ. Results of a ventricular stimulation protocol using a maximum of 4 premature stimuli in patients without documented or suspected ventricular arrhythmias. Am J Cardiol 1983; 1;52:1214-8.

40 Brugada J, Brugada R, Antzelevitch C et al. Long-term follow-up of individuals with the electrocardiographic pattern of right bundlebranch block and ST-segment elevation in precordial leads V1 to V3. Circulation 2002;105:73-8.

41 Brugada P, Brugada R, Mont L et al. Natural history of Brugada syndrome: the prognostic value of programmed electrical stimulation of the heart. J Cardiovasc Electrophysiol 2003;14:455-7.

42 Brugada J, Brugada R, Brugada P. Determinants of sudden cardiac death in individuals with the electrocardiographic pattern of Brugada syndrome and no previous cardiac arrest. Circulation 2003;108:3092-6.

43 Probst V, Veltmann C, Eckardt L et al. Long-term prognosis of patients diagnosed with Brugada syndrome: Results from the FINGER Brugada Syndrome Registry. Circulation 2010;121:635-43.

44 Letsas K.P, Liu T, Shao Q et al. Meta-analysis on risk stratification of asymptomatic individuals with the Brugada phenotype. Am J Cardiol 2015;116:98-103.

45 Sroubek J, Probst V, Mazzanti A et al. Programmed ventricular stimulation for risk stratification in the Brugada syndrome: a pooled analysis. Circulation 2016, epub ahead of print.

46 Nazarian S, Bluemke DA, Lardo AC et al. Magnetic resonance assessment of the substrate for inducible ventricular tachycardia in non-ischemic cardiomyopathy. Circulation 2005;11:2821-5.
47 Iles L, Pfluger H, Lefkovits L et al. Myocardial fibrosis predicts appropriate device therapy in patients with implantable cardioverter-defibrillators for primary prevention of sudden cardiac death. J Am Coll Cardiol 2011;57:821-8.

48 Li X, Chan CP, Hua W et al. Prognostic impact of late gadolinium enhancement by cardiac magnetic resonance imaging in patients with non-ischaemic dilated cardiomyopathy. Int J Cardiol 2013;168:4979-80.

49 Chan RH, Maron MS Maron BJ, et al. Prognostic value of quantitative contrast-enhanced cardiovascular magnetic resonance for the evaluation of sudden death risk in patients with hypertrophic cardiomyopathy. Circulation 2014;130:484-95.

50 Cluitmans MJ, Peeters RL, Westra RL, Volders PG. Non-invasive reconstruction of cardiac electrical activity: update on current methods, applications and challenges. Neth Heart J 2015:23;301-11.

51 Revishvili AS, Wissner E, Lebedev DS et al. Validation of the mapping accuracy of a novel non-invasive epicardial and endocardial electrophysiology system. Europace 2015:17:1282-8.

52 Cheng A, Zhang Y, Blasco-Colmenares E et al. Protein biomarkers identify patients unlikely to benefit from primary prevention implantable cardioverter defibrillators. Findings from the prospective observational study of implantable cardioverter defibrillators (PROSE-ICD). Circ Arrhythm Electrophysiol 2014:7:1084-91.

53 Levine YC, Rosenberg MA, Mittleman M et al. B-type natriuretic peptide is a major predictor of ventricular tachyarrhythmias. Heart Rhythm 2014: 11:1109-16.

54 Ackerman MJ, Priori SG, Willems S et al. HRS/EHRA expert consensus statement on the state of genetic testing for the channelopathies and cardiomyopathies this document was developed as a partnership between the Heart Rhythm Society (HRS) and the European Heart Rhythm Association (EHRA). Heart Rhythm 2011:8:1308-39.

55 Mullally J, Goldenberg I, Moss AJ et al. Risk of life threatening cardiac events among patients with long QT syndrome and multiple mutations. Heart Rhythm 2013:10:378-82.

56 Bezzina CR, Pazoki R, Bardai A et al. Genome-wide association study identifies a susceptibility locus at 21q21 for ventricular fibrillation in acute myocardial infarction. Nat Genet 2010;42:688-91.

57 Marsman RF, Bezzina CR, Freiberg $\mathrm{F}$ et al. Coxsackie and adenovirus receptor is a modifier of cardiac conduction and arrhythmia vulnerability in the setting of myocardial ischemia. J Am Coll Cardiol 2014;63:549-59.

58 Huertas-Vazquez A, Nelson CP, Sinsheimer JS et al. Cumulative effects of common genetic variants on risk of sudden cardiac death. Int J Cardiol Heart Vasc 2015;1:88-91.

Address for correspondence: Dr Bode Ensam, Division of Clinical Sciences, Cardiovascular Sciences Research Center, St George's University of London, London.

Email: bensam@sgul.ac.uk 